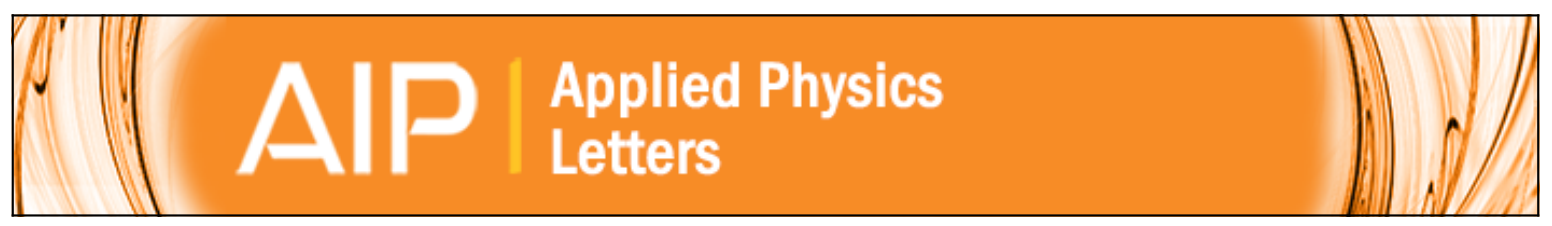

\title{
Controlled three-dimensional manipulation of vanadium oxide nanotubes with optical tweezers
}

Jose Luis Hernández-Pozos, Woei Ming Lee, Liliana Irais Vera-Robles, Antonio Campero, and Kishan Dholakia

Citation: Applied Physics Letters 93, 243107 (2008); doi: 10.1063/1.3039407

View online: http://dx.doi.org/10.1063/1.3039407

View Table of Contents: http://scitation.aip.org/content/aip/journal/apl/93/24?ver=pdfcov

Published by the AIP Publishing

\section{Articles you may be interested in}

Thermal stability and the magnetic properties of hybrid vanadium oxide-tetradecylamine nanotubes

J. Appl. Phys. 112, 053912 (2012); 10.1063/1.4749417

Ferromagnetism in water-doped vanadium oxide nanotubes

J. Appl. Phys. 108, 023905 (2010); 10.1063/1.3463425

Mechanical and electronic properties of vanadium oxide nanotubes

J. Appl. Phys. 105, 074317 (2009); 10.1063/1.3103280

Spectroscopic and microscopic study of vanadium oxide nanotubes

J. Appl. Phys. 101, 084301 (2007); 10.1063/1.2716157

Optical tweezers and confocal microscopy for simultaneous three-dimensional manipulation and imaging in concentrated colloidal dispersions

Rev. Sci. Instrum. 75, 2960 (2004); 10.1063/1.1784559

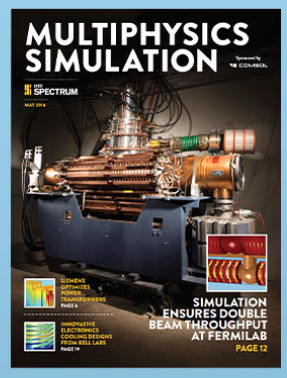




\title{
Controlled three-dimensional manipulation of vanadium oxide nanotubes with optical tweezers
}

\author{
Jose Luis Hernández-Pozos, ${ }^{1, a)}$ Woei Ming Lee, ${ }^{2, a)}$ Liliana Irais Vera-Robles, ${ }^{3}$ \\ Antonio Campero, ${ }^{3}$ and Kishan Dholakia ${ }^{2}$ \\ ${ }_{1}^{1}$ Departamento de Física, Universidad Autónoma Metropolitana-Iztapalapa (UAM-I), \\ Av. San Rafael Atlixco No. 186, Col Vicentina, C.P.09340, Mexico City, Mexico \\ ${ }^{2}$ SUPA, School of Physics and Astronomy, University of St Andrews, North Haugh, \\ St. Andrews KY16 9SS, United Kingdom \\ ${ }^{3}$ Departamento de Química, Universidad Autónoma Metropolitana-Iztapalapa, \\ P.O. Box 55-534, México DF 09340, Mexico
}

(Received 12 September 2008; accepted 23 October 2008; published online 15 December 2008)

\begin{abstract}
We present a direct nanotube-microsphere tagging technique for the controlled three-dimensional (3D) manipulation and transportation of vanadium oxide nanotubes $\left(\mathrm{VO}_{x}-\mathrm{NTs}\right)$ with optical tweezers. The high scattering and absorptive nature of the $\mathrm{VO}_{x}$-NTs preclude the $3 \mathrm{D}$ optical trapping of such nanostructures. $\mathrm{VO}_{x}$-NTs are adhered to 3-aminopropyl-triethoxysilane functionalized silica microspheres, which act as handles for indirectly manipulating and transporting the nanotubes in three dimensions with optical tweezers. The optical tweezers can also operate as optical scissors that can remove the dielectric handles and trim these nanotubes. This technique may be extended to the optical manipulation of nanotubes of any material. (C) 2008 American Institute of Physics. [DOI: 10.1063/1.3039407]
\end{abstract}

Metallic and semiconductor nanostructures, such as nanotubes (NTs), nanowires and nanorods, have immense potential for applications pertaining to nanophotonics and nanoelectronics especially due to their electronic, mechanical, and optical properties. ${ }^{1}$ Three-dimensional (3D) positional control over a single semiconductor nanostructure would be beneficial in the construction of photonics and electronics microdevices. Over the past three decades, the single beam optical tweezers ${ }^{2}$ technique has been proven versatile in many areas of physical and biological sciences. ${ }^{3}$ Typically nanometric objects of different types of materials, i.e. metallic nanoparticles, ${ }^{4}$ semiconductor nanowires, ${ }^{5-8}$ and carbon NTs, ${ }^{9}$ may be held in an optical trap. Semiconductor nanostructures exhibit a relatively high refractive index at the near infrared wavelengths which is ideal for trapping (if the high refractive index is too high, this can cause a problem). ${ }^{5}$ Multiple optical tweezers systems, e.g., holographic optical tweezers ${ }^{6}$ or time-shared optical tweezers, ${ }^{7}$ may also trap such structures in 3D. A simpler approach would be to use an elongated potential ${ }^{8}$ (a line intensity pattern) to optically manipulate a nanostructure in two dimensions. Optoelectronic tweezers ${ }^{10}$ have also shown the ability to manipulate a large number of single nanostructures simultaneously in two dimensions. Optical tweezers have shown to be able to transport and deposit bundles of single or multiwalled carbon $\mathrm{NTs}^{9}$ onto a substrate.

In recent years, multiwalled vanadium oxide $\mathrm{NTs}^{11}$ $\left(\mathrm{VO}_{x}\right.$-NTs, where $\left.x \approx 2.4\right)$ have been synthesized by the formation of lamellar structures of vanadium oxide to which amine molecules have been intercalated. When these thin layers of vanadium oxide are exposed to hydrothermal treatment, the $\mathrm{VO}_{x}$-NTs are formed as shown in Fig. 1. The formation process is described elsewhere. ${ }^{12} \mathrm{VO}_{x}$-NTs (with and without its amine molecules substituted with several

\footnotetext{
a) Authors to whom correspondence should be addressed. Electronic addresses: jlhp@xanum.uam.mx and wml6@st-andrews.ac.uk.
}

cations ${ }^{13}$ ) can be used in a number of applications, i.e., as a catalyst, ${ }^{14}$ electrodes for lithium $\left(\mathrm{Li}^{+}\right)$batteries, ${ }^{15}$ optical limiting, ${ }^{16}$ and spin control via a transition from a semiconductor to a ferromagnet. ${ }^{17}$ In general, certain NTs (e.g., vanadium oxide or carbon) may be optically absorptive which can limit and restrict their manipulation. ${ }^{9}$

In this paper, we describe a simple but powerful microsphere tagging technique to manipulate absorptive $\mathrm{VO}_{x}$-NTs. A systematic tag and removal procedure using functionalized microspheres held with a steerable optical tweezers system is shown for the efficient trapping and transportation of these NTs. The very same optical tweezers serves as a form of optical scissors that performs precise trimming of these $\mathrm{VO}_{x}$-NTs due their high optical absorption. The versatility of this optical technique allows the selective tagging, manipulation, and trimming of independent or bundles of $\mathrm{VO}_{x}$-NTs onto a chosen substrate and minimizes physical damage due to any absorption. Using two optical tweezers (one steerable and the other fixed), we are able to stably manipulate a bundle of $\mathrm{VO}_{x}$-NTs in three dimensions via tagged microspheres. We remark that an analogous dielectric microsphere

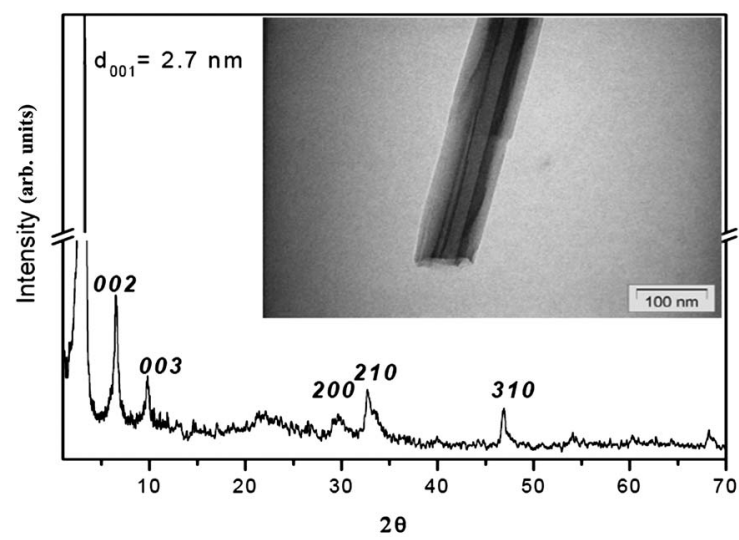

FIG. 1. X-ray diffraction pattern of the $\mathrm{VO}_{x}$-NTs (inset, the TEM image of a single $\mathrm{VO}_{x}-\mathrm{NT}$ ) 


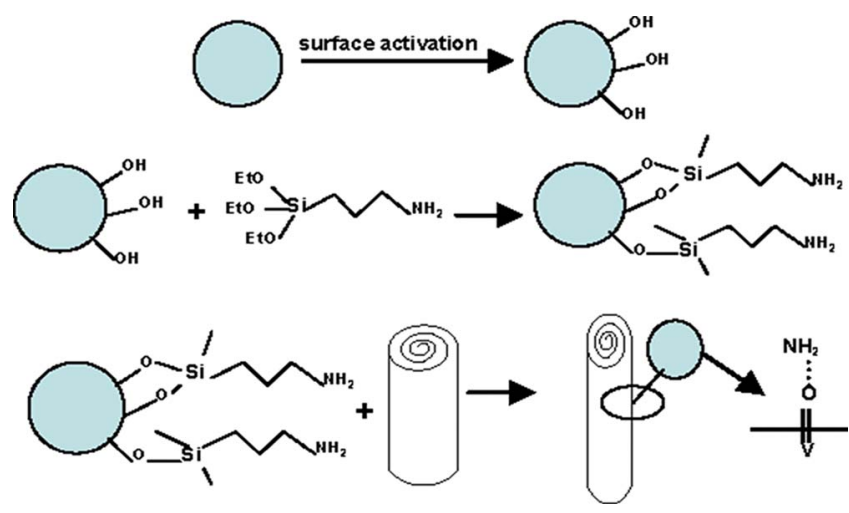

FIG. 2. (Color online) Illustration of APTES coating onto silica microspheres. Silica microspheres are cleaned to expose silanol groups and to remove any contamination from the surface. They are then functionalized with APTES; the union between the bead and the APTES is a covalent bond. Once functionalized, the amine group $\left(-\mathrm{NH}_{2}\right)$ of the APTES molecule interacts with the vanadyl groups $(\mathrm{V}=\mathrm{O})$ at the tube's surface forming a hydrogen bond (not covalent).

tagging process is employed in the optical manipulation of macromolecules ${ }^{3}$ and in the active sorting of cells through an optical potential energy landscape. ${ }^{18}$

The preparation of the $\mathrm{VO}_{x}$-NTs was carried out according to a procedure described elsewhere. ${ }^{12,13}$ The structures of the $\mathrm{VO}_{x}$-NTs are verified through the use of transmission electron microscopy (TEM) imaging and $x$-ray diffraction. In Fig. 1, we show the x-ray diffraction pattern of $\mathrm{VO}_{x}$-NTs and the TEM image of a single $\mathrm{VO}_{x}-\mathrm{NT}$ (inset). From the $\mathrm{x}$-ray diffraction pattern, it is possible to see three reflections at low angle (lattice structure indexed here as 001, 002, and 003). These reflections indicate the layered structure of the walls within the $\mathrm{VO}_{x}$-NTs. In this case the position of the first peak corresponds to an interlayer distance of $2.7 \mathrm{~nm}$. At higher angles, the peaks indexed as $h k 0$ correspond to the crystalline structure of the walls. The walls consist of layers of $\mathrm{V}_{7} \mathrm{O}_{16}$ formed by two sheets of $\mathrm{VO}_{5}$ square pyramids pointing in opposite directions, which are joined by $\mathrm{VO}_{4}$ in the form of a tetrahedron. X-ray diffraction patterns were taken with a Siemens D-500 diffractometer using the $K \alpha$ line $(\lambda=1.54 \AA)$. Micrographs were taken with a Carl-Zeiss EM-910 transmission electron microscope operated at 120 $\mathrm{kV}$. Typically, the thickness of these $\mathrm{VO}_{x}$-NTs ranges from around 20 to $30 \mathrm{~nm}$; the tube diameter varies between 50 and $120 \mathrm{~nm}$ and their lengths between 0.5 and $4 \mu \mathrm{m}$. The tube diameter will vary $(\sim 0.5-1 \mu \mathrm{m})$ with respect to the degree of unfolding that it may exhibit over time. ${ }^{19}$

Next we describe the functionalization of silica microspheres $(2.7 \mu \mathrm{m}$ in diameter, Bangs Laboratories) by using 3-aminopropyl-triethoxysilane (APTES). ${ }^{20}$ These attach to the $\mathrm{VO}_{x}$-NTs as they possess amine groups $\left(-\mathrm{NH}_{2}\right)$, which have affinity toward the $\mathrm{VO}_{x}$-NTs surface via van der Waals interactions (see Fig. 2). The functionalized silica microspheres were suspended in de-ionized water $(1 \mathrm{ml})$, while the $\mathrm{VO}_{x}$-NTs were suspended in an aqueous solution $(1 \mathrm{ml})$ containing a mixture of $70 \%$ ethanol and $30 \%$ de-ionized water. Both samples were sonicated before they were mixed together in an enclosed chamber as described elsewhere. ${ }^{21}$

The experiment used for the trapping studies is adopted from the standard dual beam optical tweezers system that was reported by Lee et al. ${ }^{21}$ The system comprises of two optical tweezers both operating at the wavelength of 1070 nm. one of which is steerable in both $x$ and $y$ using a dual

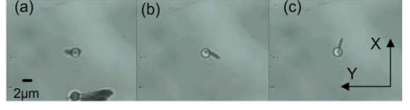

(d)
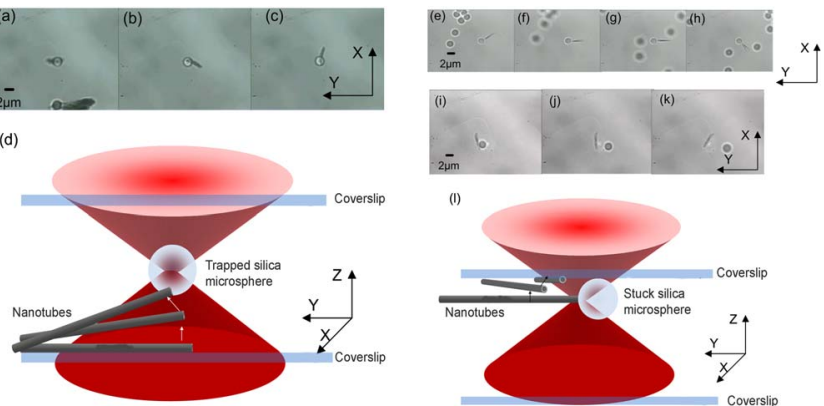

FIG. 3. (Color online) Illustration of tagging and untagging procedure of the NT to a trapped coated silica microsphere. (a)-(c) show a single NT being picked up by a trapped functionalized dielectric silica microsphere. (d) shows a $3 \mathrm{D}$ illustration of the entire tagging illustration of the entire tagging process. (e)-(h) show a large NT transported in both the lateral and the axial planes by a trapped silica microsphere. (i)-(k) show the experiment results of the controlled removal of the NTs from the dielectric microsphere. (1) shows a $3 \mathrm{D}$ illustration of this removal process.

axis acousto-optical deflector while the second optical tweezers are set at a fixed position.

In what follows, we illustrate the experimental results from the manipulation of $\mathrm{VO}_{x}$-NTs with the steerable optical tweezers. First, we show how to controllably tag the microsphere with the NTs (Fig. 3). Here a single optically trapped APTES-coated silica sphere is carefully positioned approximately $5 \mu \mathrm{m}$ above a single $\mathrm{VO}_{x}$-NT (length of $\sim 5 \mu \mathrm{m}$, diameter of $\sim 1 \mu \mathrm{m}$ ). The slightly large diameter of this tube indicates a minor unrolling of the tubular structure. ${ }^{19}$ The NT is observed to be pulled toward the focused light beam. Upon contact, the NT is seen to attach itself onto the microsphere as seen in Figs. 3(a) and 3(b). Figure 3(c) shows that the microsphere with the tagged NT is being optically steered away as indicated by the orientation of the NT. Figure 3(d) shows a graphical illustration of how the NT are attached to the silica microsphere. Figures 3(e)-3(h) show that a NT (length of $\sim 5 \mu \mathrm{m}$, diameter of $\sim 0.5 \mu \mathrm{m}$ ) is being transported around the sample by the optically trapped silica microsphere at velocities of up to $20 \mu \mathrm{m} / \mathrm{s}$. Importantly, this form of tagging process is completely reversible. In Figs. 3(i)-3(k), we show a single silica sphere with an attached NT (length of approximately $5 \mu \mathrm{m}$ and diameter of around $0.5 \mu \mathrm{m})$ deposited onto the top cover slip. By carefully scanning the optical tweezers $(<10 \mathrm{~mW})$ across the junction where the NT is attached to the microsphere, the NT is detached from the microsphere. Figure 3(1) shows a graphical illustration of this removal process at the top cover slip.

In Fig. 4, we show a series of images that depict the manipulation of the NTs in three dimensions. In Figs. 4(a)-4(d), we show the two functions in which the steerable optical tweezers serve both as the trapping beam and the optical scissors. A cluster of NTs tagged to a single silica microsphere is positioned around $2 \mu \mathrm{m}$ above the bottom cover slip [Fig. 4(a)]. By swiftly scanning the tweezing beam across a small section of the cluster and back onto the microsphere, a small section of a NT (length: $2 \mu \mathrm{m}$, width: $0.5 \mu \mathrm{m})$ is trimmed and removed from the large cluster [Fig. 4(b)]. As the tweezing beam is scanned back onto the microsphere, the trimmed section of the NT is reattached onto the optically trapped silica microsphere [Figs. 4(c) and 4(d)].

Figures 4(e)-4(h) show the manipulation of a single NT (length: $5 \mu \mathrm{m}$, diameter: $0.5 \mu \mathrm{m}$ ) where one end of the NT is anchored onto a cluster of silica microsphere at the bottom 


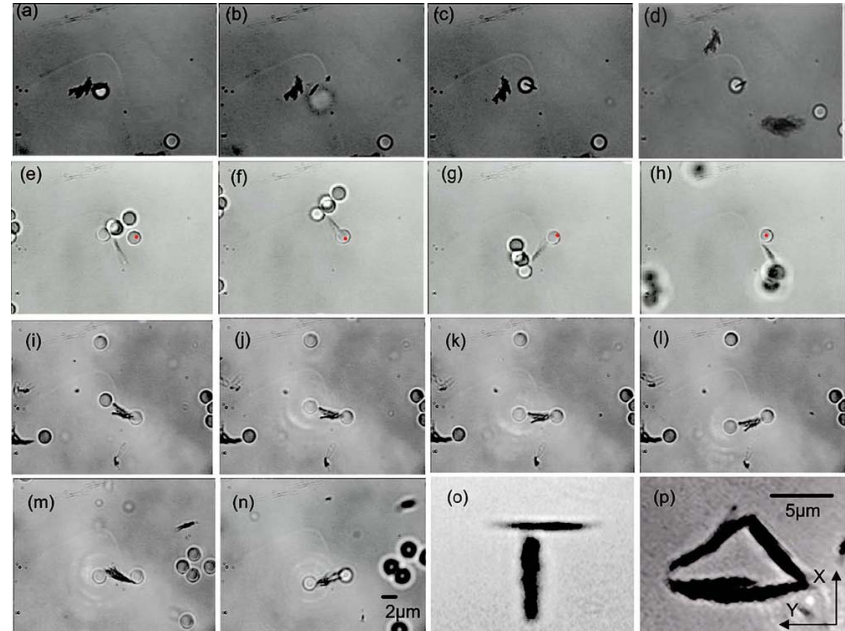

FIG. 4. (Color online) A series of images depicting the manipulation of the NTs in three dimensions. (a)-(d) show selective trapping and trimming of a single NT from a cluster of NTs and subsequent tagging to an optically trapped single silica microsphere. (e)-(h) show the manipulation of a single NT over the bottom cover slip. [(i)-(n)] Rotation and controlled levitation of a bundle of NTs held with two optical tweezers via the two microspheres tagged onto opposite ends of the bundle of NTs. (o) and (p) show the two-dimensional patterning of the NTs into a T-shaped and a triangle, respectively.

cover slip. An optically trapped microsphere (marked by a red dot) is moved onto the free end of the NTs for attachment [Fig. 4(f)]. Such an optical tweezing configuration allows a 3D angular tilt position of the NT over a substrate of interest.

In Figs. 4(i)-4(1), we now employ two optical tweezers to demonstrate controlled rotation, at angular increments of $10^{\circ}$, of a bundle of NTs with two microspheres tagged onto opposite ends of the bundle. The steerable optical tweezers move the position of one of the microspheres (laterally translated at steps of $\sim 1 \mu \mathrm{m}$ ), while the fixed optical tweezers hold the other microsphere at a fixed position. This shows the angular positioning of the bundle of NTs with this technique. By shifting the axial position of two trapped microspheres away from the bottom of the cover slip by $5 \mu \mathrm{m}$, the bundle of NTs is stably lifted off the bottom cover slip as seen in Figs. 4(m) and 4(n). Next, we transported and deposited a bundle of NTs onto the top cover slip. By using the trapping beam as optical scissors, we were able to trim small piece of the tubes and thereafter position them into a desired pattern (see also Plewa et al. ${ }^{9}$ ). In Figs. 4(o) and 4(p), we show the controlled two-dimensional patterning of the NTs by single beam optical tweezers into T-shape and triangle, respectively. The fine positioning of the NTs into its desired shape is done by carefully steering a slightly defocused beam at the perimeter of the NTs. In this way, the NTs are seen to be gently pushed away (due to scattering) from the beam without being ablated.

In conclusion, we have qualitatively demonstrated the method of dielectric tagging of NTs for optical micromanipulation. Our experiments show that this approach is both feasible and controllable for absorptive NTs. The indirect manipulation and positioning of NTs via tagging by an optically trapped microsphere can be performed in all three dimensions. This is an improvement from more direct optical trapping ${ }^{9}$ as it potentially offers more controllability and obviates any issues due to heating. This approach can be extended to the manipulation and transportation of nanostructures of other material compositions. Further employment of specific surface chemistry upon microspheres may lead to an active optical sorting of NTs through an optical potential energy landscape. ${ }^{18}$

The authors would like to thank Pascal Andre for use of equipment for functionalization process. L. I. Vera-Robles would like to thank the Mexican Consejo Nacional de Ciencia y Tecnología (CONACyT) for the scholarship. J. L. Hernández-Pozos acknowledges the support from the Academia Mexicana de Ciencias and the Royal Society (U.K.). K.D. is a Royal Society-Wolfson Merit Award holder. We thank the EPSRC for funding.

${ }^{1}$ Y. Cui and C. M. Lieber, Science 291, 851 (2001).

${ }^{2}$ A. Ashkin, J. M. Dziedzic, J. E. Bjorkholm, and S. Chu, Opt. Lett. 11, 288 (1986).

${ }^{3}$ K. Dholakia and P. Reece, Nanotoday 1, 18 (2006).

${ }^{4}$ P. M. Hansen, V. K. Bhatia, N. Harrit, and L. Oddershede, Nano Lett. 5, 1937 (2005)

${ }^{5}$ P. J. Pauzauskie, A. Radenovic, R. Trepagnier, H. Shroff, P. Yang, and J. Liphardt, Nature Mater. 5, 97 (2006).

${ }^{6}$ R. Agarwal, K. Ladavac, Y. Roichman, G. H. Yu, C. M. Lieber, and D. G. Grier, Opt. Express 13, 8906 (2005).

${ }^{7}$ A. van der Horst, A. I. Campbell, L. K. van Vugt, D. A. M. Vanmaekelbergh, M. Dogterom, and A. van Blaaderen, Opt. Express 15, 11629 (2007).

${ }^{8}$ T. Yu, F. C. Cheong, and C. H. Sow, Nanotechnology 15, 1732 (2004).

${ }^{9}$ J. Plewa, E. Tanner, D. M. Mueth, and D. G. Grier, Opt. Express 12, 1978 (2004); S. D. Tan, H. A. Lopez, C. W. Cai, and Y. G. Zhang, Nano Lett. 4, 1415 (2004).

${ }^{10}$ J. A. Rogers, Nat. Photonics 2, 69 (2008).

${ }^{11}$ M. E. Spahr, P. Bitteli, R. Nesper, M. Müller, F. Krumeich, and U. Nissen, Angew. Chem., Int. Ed. 37, 1263 (1998).

${ }^{12}$ L. I. Vera-Robles and A. Campero, "A novel approach to vanadium oxide nanotubes by oxidations of V4+ species," J. Phys. Chem. C (in press).

${ }^{13}$ L. I. Vera-Robles, F. U. Naab, A. Campero, J. L. Duggan, and F. D. McDaniel, Nucl. Instrum. Methods Phys. Res. B 261, 534 (2007).

${ }^{14}$ B. Azambre, M. J. Hudson, and O. Heintz, J. Mater. Chem. 13, 385 (2003).

${ }^{15}$ A. Dobley, K. Ngala, S. F. Yang, P. Y. Zavalij, and M. S. Whittingham, Chem. Mater. 13, 4382 (2001).

${ }^{16}$ J. F. Xu, R. Czerw, S. Webster, D. L. Carroll, J. Ballato, and R. Nesper, Appl. Phys. Lett. 81, 1711 (2002).

${ }^{17}$ L. Krusin-Elbaum, D. M. Newns, H. Zeng, V. Derycke, J. Z. Sun, and R. Sandstrom, Nature (London) 431, 672 (2004).

${ }^{18}$ K. Dholakia, W. M. Lee, L. Paterson, M. P. MacDonald, R. McDonald, I. Andreev, P. Mthunzi, C. T. A. Brown, R. F. Marchington, and A. C. Riches, IEEE J. Sel. Top. Quantum Electron. 13, 1646 (2007).

${ }^{19}$ S. Webster, R. Czerw, R. Nesper, J. DiMaio, J. F. Xu, J. Ballato, and D. L. Carroll, J. Nanosci. Nanotechnol. 4, 260 (2004).

${ }^{20}$ M. Halliwell and A. E. G. Cass, Anal. Chem. 73, 2476 (2001).

${ }^{21}$ W. M. Lee, P. J. Reece, R. F. Marchington, N. K. Metzger, and K. Dholakia, Nat. Protoc. 2, 3226 (2007). 